\title{
Descriptions of four larval forms of Nilodosis Kieffer from East Asia
}

\author{
Hongqu Tangl and Masaru Yamamoto 2
}

\begin{abstract}
Tang H and Yamamoto M. 2012. Descriptions of four larval forms of Nilodosis Kieffer from East Asia. Fauna norvegica 31: 205-213.

Larval material putatively assigned to the genus Nilodosis Kieffer from Korea, China and Japan has been compared. The results show that the Japanese larval form has the club- to balloon-shaped cephalic setae S7 and S9 in common with the Korean larval form, but it can be separated from the latter by the shape of the inner mandibular teeth and the premandibular teeth. The larval forms from China (Guangdong and Yunnan) apparently consist of two independent species. It is most likely that there will be more species in this genus found in Asia. Larvae are mud-sandy bottom-dwellers that can occur in the littoral of lakes and the potamal of larger rivers, up to a maximum depth of 5 meters. The specific larval characters show that it probably is a semi-psammorheophilic predator.
\end{abstract}

doi: 10.5324/fn.v31i0.1406. Received: 2011-11-05. Accepted: 2012-06-28.

Published on paper and online: 2012-10-17.

Keywords: Nilodosis, East Asia, larva, dweller

1. Jinan University, Research Centre of Hydrobiology, Guangzhou 510632, P.R. China

2. 1-6-12, Satomachi, Yoshimi, Shimonoseki, Yamaguchi Prefecture, 759-6525, Japan

Corresponding author: Hongqu Tang

E-mail:townningt@gmail.com

\section{INTRODUCTION}

The genus Nilodosis Kieffer was described first in 1921 based on Sudanese (Afrotropical) material, but information on this genus remains sparse and insufficient. Until now, only two species were reported, N. fusca Kieffer and N. grisea Freeman, both of which are from the Afrotropics. The genus can be separated in the adult stage from others by the arcuate curved long tibial spur on the foreleg, absence of pulvilli, absence of an apical strong long seta on the inferior volsella, the superior volsella either with expanded setose and microtrichiose basal pad, bearing elongate, bare, apically hooked digitus (projection) (N. fusca) or solely a triangular setose and microtrichiose lobe, without digitus (N. grisea) in the male. Tergite IX of the female is extremely large in Nilodosis, extending posterior to the cerci (Cranston \& Spies, 1999).

The larva of $N$. fusca is characterized by having the cleft mentum shared with the genus Fissimentum Cranston \& Nolte. However, Nilodosis can be distinguished from the latter by the following features: 1) lacking any indication of Lauterborn organs; 2) clustered inner teeth of the mandible; 3 ) pecten epipharyngis consisting of toothed scales ( $N$. fusca) or single scale ( $N$. sp. Japan and $N$. sp. Korea); 4) frontal seta S4 reduced to a peg (N. fusca) or normal (Asian forms in this paper); 5) maxilla includes a cluster of chaetulae dorsal to the palpiger (Cranston \& Spies, 1999). In addition to the type localities in Africa, the genus was presumed also to occur in Brazil, Australia and Southeast Asia based on unreared larvae (Cranston \& Spies, 1999; Cranston, pers. comm.). However, information from East Asia about this genus is very poor. With regard to the Japanese material, Kitagawa (1997) identified larvae collected from Kiso River, Aichi Prefecture and Lake Biwa, Shiga Prefecture, to Chironominae Genus CA, and included a brief description and detailed figures. Later, the Chironominae Genus CA specimens were re-identified as Fissimentum sp. FA by Kitagawa (2001). A similar larva identified as Fissimentum sp. collected from Lake Towada, Akita Prefecture was reported by Ueno et al. (2001). These larvae reported by Kitagawa and Ueno et al. were confirmed to be conspecific, and putatively identified as "Nilodosis" by the junior author in this paper rather than belonging to the genus Fissimentum.

During a project on water biomonitoring in East Asia more than 13 larvae were found from Korea and China by the senior author. Although the detailed structures are different, the basic structures coincide with Japanese material. Thus, these larvae 
are putatively also identified as Nilodosis. Four morphologically different larvae are described below. However, due to the lack of associated larval/pupal/adult material, the work on the taxonomy of these taxa of "Nilodosis" is incomplete and the assignment to Nilodosis remains tentative.

\section{MATERIALS AND METHODS}

In total, seven sites were studied, including one site from Korea (Figure 1A), two sites from China (Figure 1 B \& C), and four sites from Japan (Figure 1 D - G). Chironomid larvae were collected by various methods, e.g., hand nets in Yunnan material, Peterson grab in Guangdong material and Ponar grab or D-frame net in Korean material. The habitats range from large rivers to lakes.

All material was mounted in Euparal, following Pinder \& Reiss (1983) and Epler (2001). Morphological terminology and abbreviations follow Sæther (1980). Measurements are given as ranges, generally followed by means when 3 or more measurements were made, followed by the number of specimens measured (n) in parentheses. Some images of larval specimens were acquired with a high-resolution Leica digital camera connected to a Zeiss microscope. Line drawings were made by using a drawing tube on an Olympus BX43.

Chinese material is deposited in the Long Term Ecology Laboratory Research Centre of Hydrobiology, Jinan University, Guangdong. Korean material is deposited in the Ecology and Behavior Laboratory, Pusan National University, Pusan. Five larvae of Japanese material were loaned from N. Kitagawa, and the remaining Japanese material is preserved in personal collection of M. Yamamoto.

\section{Taxonomic Result}

\section{Nilodosis Kieffer}

Nilodosis Kieffer, 1921: 30; 1922: 48.

Type-species: Nilodosis fusca Kieffer, 1922 by subsequent monotypy (Kieffer, 1922: 48); Freeman, 1957b: 407; Cranston and Spies, 1999: 249.

For all previous records, see Table 1 .

\section{Diagnosis}

For larvae; emended assuming that the larval forms described below belong to Nilodosis.

Medium to large, up to $13 \mathrm{~mm}$ in length, red color in living material. Head capsule brown or yellow-brown, beanshaped in lateral view, eye spots oblique posterolaterally. Posterior occipital margin dark, heavily sclerotized, with a wide triangulum occipitale (Figure 2).

Dorsal surface of head (Figure 4A, 7A): Frons present. Clypeal sclerite absent ( $N$. sp. Korea) or reduced ( $N$. fusca). Labral sclerite variable. The insertion and shape of some cephalic setae varies, e.g., frontal seta 4 (S4) reduced in N. fusca. Suborbital seta (S6) and supraorbital seta (S7) adjacent to each other and

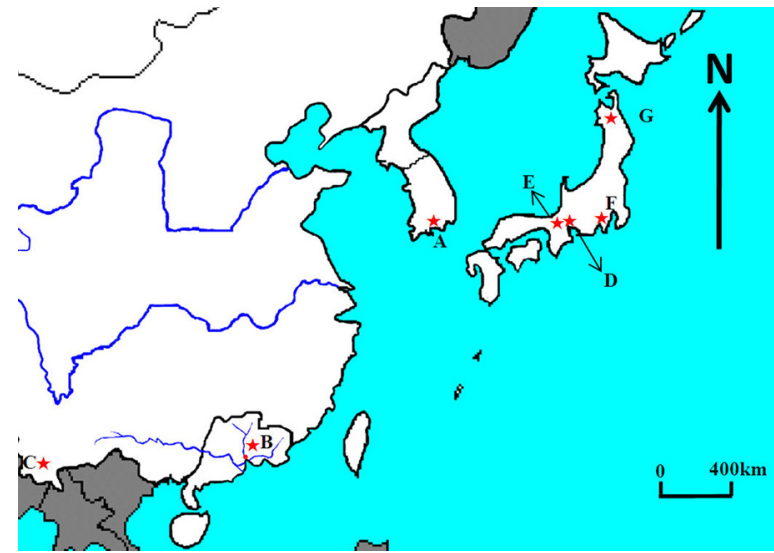

Figure I. Locations of the "Nilodosis" larvae occurring in East Asia. A) Jingyang lake, Jinju, South Korea; B) Luitian river, Guangzhou, Guangdong province, China; C) Mekong river, Xishuangbanna, Yunnan province, China; D) Kiso River, downstream of Noubi bridge, Aichi Prefecture (Kitagawa, 1997); E) ;Yasu River, inlet of Biwa Lake, Shiga Prefecture, Japan (Kitagawa, 1997); F) Motosu Lake, Yamanashi Prefecture, Japan (Kitagawa, 1997); G) Towada Lake, Akita Prefecture (Ueno et al., 2001).

S7 \& genal seta 9 (S9) rather short balloon-shaped in "N." sp. Japan and "N." sp. Korea.

Antenna: 6-segmented, Lauterborn organ absent. AR less than 1.0, blade usually distinctly longer than flagellum.

Labro-epipharynx: SI large, plumose, basally contiguous or not, outer margin with fine serrations. SII and SIII simple. Labral lamellae broad, well developed, without indication of median division, distal margin with 15-20 nearly even teeth. Pecten epipharyngis of three separated scales or only one plate in the Japan and Korea specimen; if 3 scales, each scale with 2-4 teeth, if only one plate, 2-3 toothed distally. Premandible with 2 apical teeth and 0-2 small inner teeth ( $N$. fusca only having 2 apical teeth), the most proximal teeth may be indistinct. Premandibular brush weak or absent.

Mandible: Strongly curved apical tooth, about equal in length to rest of mandible. Dorsal tooth absent. Inner tooth either with one distinct large tooth associated with 1-3 small teeth (Asia larval form) or three clumped (N. fusca). Seta subdentalis thick, extending along the inner margin of apical tooth, not extending beyond the tip. Pecten mandibularis absent.

Mentum: Characteristically with V-shaped cleft mentum, each side with 7-8 teeth, anterolaterals most protruding. Innermost first or second pair of teeth usually paler than rest. Ventromental plates elongated, acutely produced median ends opposite to each other, not extending to border of mentum cleft. Striae distinct in middle, usually with hyaline posterior lobes. Distance between setae submenti greater than the width of dorsomentum.

Maxilla: One or two cluster of chaetulae well developed dorsal to maxillary palp. Antaxial seta and lacinial chaetae slender and well developed. Apical seta of palp at least longer than the half length of palp. 


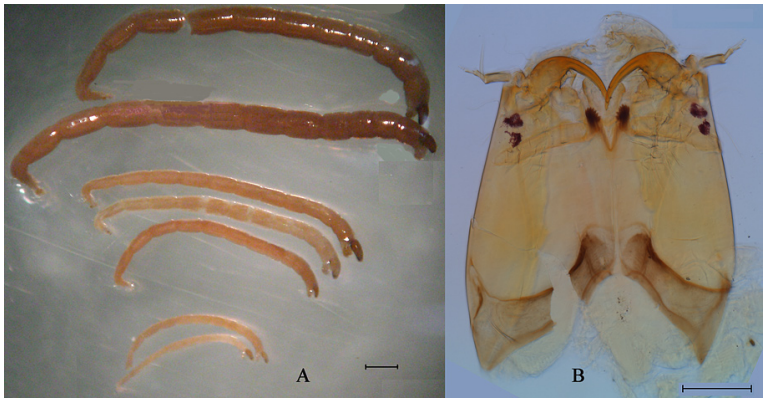

Figure 2. Photos of different "Nilodosis" instars (A, Korean material, scale $=1 \mathrm{~mm})$ and ventral view of head capsule $(B$, Guangdong material, scale $=100 \mu \mathrm{m})$.

Body: Posterior parapods with 14-16 claws. Two pairs of anal tubules present. Procercus bearing 6-7 anal setae, supraanal setae long and strong, located on the anterior of anal tubules.

\section{Descriptions}

\section{“Nilodosis" sp. Korea}

(Figure 2-A, Figure 3, Figure 4)

Material examined: A total of 11 larvae were examined first in alcohol. Seven larvae subsequently were cleared and mounted on slides, including three $4^{\text {th }}$, one $3^{\text {rd }}$ and three $2^{\text {nd }}$ instars. South

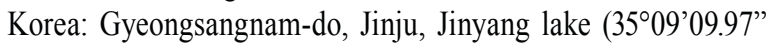
N, 12759'41.46” E), 13. ix. 2009, leg. Woon-Seok Cho.

\section{Fourth instar (n=3)}

Total length 9.8-12.6, $11.3 \mathrm{~mm}$. Head capsule dark brown, 620640, $630 \mu \mathrm{m}$ long, 350-380, $365 \mu \mathrm{m}$ wide. Postmentum length $415-420,418 \mu \mathrm{m}$. Triangulum occipitale as in generic diagnosis, with 2 distinct broad areas in the caudolateral corner enclosed by the clear dark traces (Figure 4B). Cephalic setae S7 and S9 short balloon-shaped. Dorsal surface of larval head as Figure $4 \mathrm{~A}$.

Antenna(Figure 3A): Total length 120-140, $135 \mu \mathrm{m}$. Flagellomere lengths (in $\mu \mathrm{m}$ ): 58.0-62.5, 61.0; 27.5-30.0, 29.2; 20.0-22.5, 22.0; $5-5,5 ; 5-5,5 ; 2.5-2.5,2.5$; AR 0.80-0.89, 0.83; first segment 2.8-3.4, 3.1 times as long as basal width, ring organ located near middle, $0.50-0.63,0.58$ times first segment from the base. Blade 80-100, $93 \mu \mathrm{m}$ long, 1.24-1.30, 1.28 times as long as flagellum. Style 6.0-7.5, $7.0 \mu \mathrm{m}$ long, located at the apex of third segment, extending to the apex of fourth segment.

Labro-epipharynx: SI distal slender plumose. Labral lamella plumose, distal margin with 18-20 teeth (Figure 3B). Pecten epipharyngis of one toothed scale. Premandible 52-55, $54 \mu \mathrm{m}$ long, with 2 distal and 2 small inner teeth, the inner 2 teeth about equal in size. Premandibular brush absent (Figure 3C).

Mandible (Figure 3D): Total length 175-180, $178 \mu \mathrm{m}$, with 1-2 small triangular inner teeth and one curved apical tooth. Apical tooth predominant, 100.0-162.5, $127.5 \mu \mathrm{m}$ long, about the same as the basal part. Seta subdentalis $60-70,68 \mu \mathrm{m}$ long, not extending the margin of apical tooth.

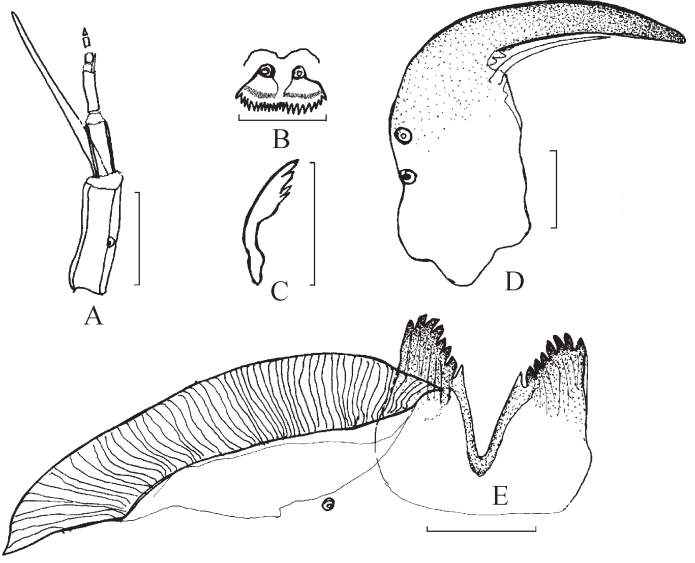

Figure 3. Larval morphology of "Nilodosis" sp. Korea. A) Antenna; B) Labral setae SI and labral lamella; C) Premandible; D) Mandible; E) Mentum (scale $=50 \mu \mathrm{m}$ ).

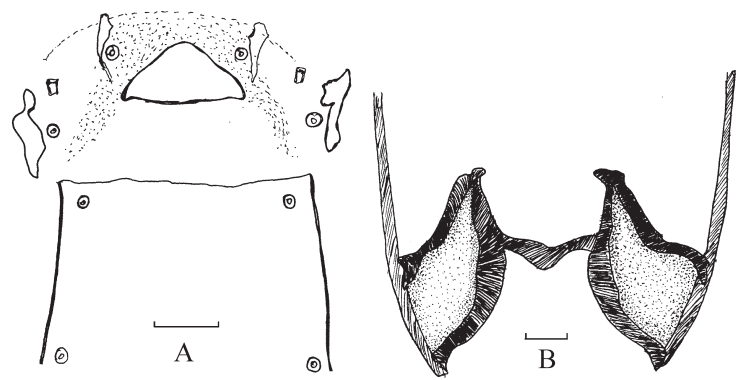

Figure 4. Larval morphology of "Nilodosis" sp. Korea. A) Dorsal surface of larval head; B) Occipital margin area and triangulum occipitale (scale $=50 \mu \mathrm{m})$.
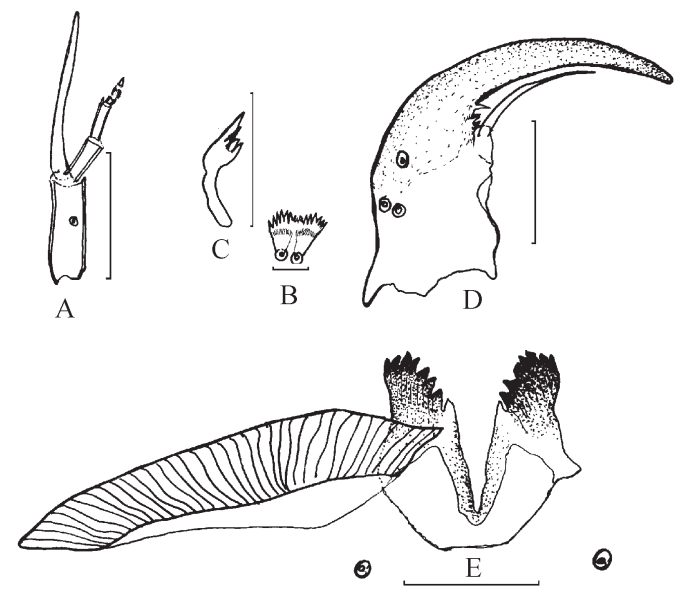

Figure 5. Larval morphology of "Nilodosis" sp. Guangdong. A) Antenna; B) Labral setae SI and labral lamella; C) Premandible; D) Mandible; E) Mentum (Scale of Figure 5B is $10 \mu \mathrm{m}$, other scale $=50 \mu \mathrm{m})$. 
Table I. The previous records of Nilodosis in the world including the records presented in this paper. The letters M, F, L, P represent male, female, larva and pupa.

\begin{tabular}{|c|c|c|c|c|c|}
\hline Species & Region & Country & Site & Stage & References \\
\hline N. fusca & N. Africa & Sudan & Shambe & M & Kieffer, 1921; Cranston \& Spies, 1999 \\
\hline N. fusca & C. Africa & Congo & Ituri & M & Goetghebuer, 1936; Cranston \& Spies, 1999 \\
\hline N. fusca & W. Africa & Burkina Faso & Red Volta & M & Freeman, 1957b; Cranston \& Spies, 1999 \\
\hline N. fusca & W. Africa & Nigeria & Eket & M & Freeman, 1957b; Cranston \& Spies, 1999 \\
\hline N. fusca & N. Africa & Sudan & Liednum & $\mathrm{M}, \mathrm{F}$ & Freeman, 1957b; Cranston \& Spies, 1999 \\
\hline N. fusca & E. Africa & Uganda & Busungwe Bay & M & Freeman, 1957b; Cranston \& Spies, 1999 \\
\hline N. fusca & C. Africa & Congo & Basoko & M & Freeman, 1957b; Cranston \& Spies, 1999 \\
\hline N. grisea & C. Africa & Congo & Lake Tanganika & M & Freeman, 1957a, b; Cranston \& Spies, 1999 \\
\hline$N . \mathrm{sp}$ & E. Africa & Uganda & Lake Albert & $\mathrm{L}$ & Chrispeels, 1959; Cranston \& Spies, 1999 \\
\hline N. fusca & S. Africa & Zimbabwe & Lake Kariba & $\mathrm{L}$ & McLachlan, 1969; Cranston \& Spies, 1999 \\
\hline N. fusca & W. Africa & Nigeria & Lake Opi & $\mathrm{F}, \mathrm{L}, \mathrm{P}$ & Hare \& Carter, 1987; Cranston \& Spies, 1999 \\
\hline$N . \mathrm{sp}$ & S. America & Brazil & Sao Paulo & $\mathrm{L}$ & Cranston \& Nolte, 1996 \\
\hline$N . \mathrm{sp}$ & Oceania & Australia & Victoria/Queensland & $\mathrm{L}$ & Cranston \& Nolte, 1996 \\
\hline N. sp & E. Africa & Tanzania & Lake Tanganika & subfossil & Eggermont \& Verschuren, 2003 \\
\hline$N . \mathrm{sp}$ & E. Asia & Japan & Kiso and Yasu River & $\mathrm{L}$ & Kitagawa, 1997 \\
\hline N. sp & E. Asia & Japan & Towada Lake & $\mathrm{L}$ & Ueno et al., 2001 \\
\hline N. sp & E. Asia & China & Yunnan & $\mathrm{L}$ & pers., in this paper \\
\hline$N . \mathrm{sp}$ & E. Asia & China & Guangdong & $\mathrm{L}$ & pers., in this paper \\
\hline N. sp & E. Asia & South Korea & Jinyang Lake & $\mathrm{L}$ & pers., in this paper \\
\hline
\end{tabular}

Mentum (Figure 3E): 85-90, $87 \mu \mathrm{m}$ wide, median cleft depth 75-80, $78 \mu \mathrm{m}$, each side with 8 teeth, innermost teeth paler than others. Ventromentum 200-225, $208 \mu \mathrm{m}$ wide, ventromentum plate ratio 2.95-3.08, 3.05. Inter-plate distance (IPD) 45-50, $48 \mu \mathrm{m}$ long. Seta submenti posterior to the mentum, distance between setae submenti 135-150, $145 \mu \mathrm{m}$ long.

Body: Procercus 40-45, $43 \mu \mathrm{m}$ high, about 2 times as long as middle width. 7 anal setae, 500-600, $568 \mu \mathrm{m}$ long, supraanal seta 350-540, $446 \mu \mathrm{m}$ long. 2 pairs of anal tubules, upper pairs 140-160, $150 \mu \mathrm{m}$ long, about 2.5-3.0, 2.8 times as long as basal width; lower pairs 220-350, $285 \mu \mathrm{m}$ long, 2.91-3.67, 3.30 times as long as basal width.

Third instar $(\mathrm{n}=1)$

Total length $8.2 \mathrm{~mm}$ (Figure 2A), with body length $7.6 \mathrm{~mm}$, head $560 \mu \mathrm{m}$ long, postmentum $350 \mu \mathrm{m}$ long.

Antenna: Total length $112.5 \mu \mathrm{m}$, flagellomere lengths (in $\mu \mathrm{m}$ ): 50.0-52.5, 52.0; 25.0-27.5, 26.0; 15.0-17.5, 16.0; 5-5, 5; 5-5, 5; 2.5$2.5,2.5$, AR 0.79. Basal segment 2.5 times as long as the basal width. Blade $75 \mu \mathrm{m}$ long, 1.12 times as long as flagellum.

Labro-epipharynx: Premandible $50 \mu \mathrm{m}$ long.

Mandible. Total length $150 \mu \mathrm{m}$, with apical tooth $100 \mu \mathrm{m}$ long. Mentum: $70 \mu \mathrm{m}$ wide, with median cleft depth $65 \mu \mathrm{m}$. Ventromentum $162.5 \mu \mathrm{m}$ wide, 2.95 times as long as the height. Inter-plates distance $40 \mu \mathrm{m}$ wide. Distance between setae submenti $120 \mu \mathrm{m}$.
Body: Anal seta 480-500 $\mu \mathrm{m}$ long, supraanal seta 360-400 $\mu \mathrm{m}$ long. The upper pair tubules $120 \mu \mathrm{m}$ long, the lower pair tubules $200 \mu \mathrm{m}$ long.

Second instar $(n=3)$

Total length 4.8-6.5, 5.7mm (Figure 2A), with body length 4.56.1, 5.4 mm, head 300-340, $323 \mu \mathrm{m}$ long, postmentum 180-190, $184 \mu \mathrm{m}$ long.

Antenna: Total length $68-75,72 \mu \mathrm{m}$, flagellomere lengths (in $\mu \mathrm{m}): 27.5-30.0,28.5 ; 15-15,15 ; 10.0-12.5,12.0 ; 2.5-3.0,2.8$; 2.5-2.5, 2.5; 1.25-1.5.0, 1.3.0, AR 0.67-0.75, 0.71. Basal segment 3.0 times as long as the basal width. Blade 48-55, $53.5 \mu \mathrm{m}$ long, 1.20-1.24, 1.22 times as long as flagellum.

Labro-epipharynx: Premandible 25-28, $26 \mu \mathrm{m}$ long. Mandible: Total length 80.0-87.5, 85.0 $\mu \mathrm{m}$, with apical tooth 50$55,53 \mu \mathrm{m}$ long, seta subdentalis $30-35,32 \mu \mathrm{m}$ long.

Mentum: 42.5-50.0, $45.8 \mu \mathrm{m}$ wide, with median cleft depth 40$45,42 \mu \mathrm{m}$. Ventromentum 94-106, $100 \mu \mathrm{m}$ wide, $2.50-2.74,2.64$ times as long as the height. Inter-plate distance 25.0-30.0, 26.7 $\mu \mathrm{m}$ wide. Distance between setae submenti $75 \mu \mathrm{m}$.

Body: Anal seta 320-340, $335 \mu \mathrm{m}$ long, supraanal seta 180-235, $208 \mu \mathrm{m}$ long. The upper pair tubules $90-100,95 \mu \mathrm{m}$ long, the lower pair tubules 120-150, $142 \mu \mathrm{m}$ long.

Remarks. The Korean larval form is similar to the Japanese population (see below), while, some differences can be observed in the mandible and premandible. The inner mandible teeth in 
the former are usually triangular, while truncated in the latter form. The most proximal inner tooth of premandible is about same size as the neighboring teeth in the former, while minute or difficult to discern in the Japanese larval form.

\section{"Nilodosis" sp. Guangdong}

(Figure 2-B, Figure 4-A, Figure 5)

Material examined: 1 larva, China, Guangdong Province, Guangzhou, Conghua, Luitian River, 15. x. 2009 (2347'34.54" N, 11350'38.23” E), leg. H.Q. Tang.

Total length $8.8 \mathrm{~mm}$. Head capsule pale brown, $500 \mu \mathrm{m}$ long, $375 \mu \mathrm{m}$ wide. Postmentum length $232.5 \mu \mathrm{m}$. Triangulum occipitale as in generic diagnosis, with 2 distinct broad areas in the caudolateral corner enclosed by the clear dark traces.

Antenna (Figure 5A): Total length $75 \mu \mathrm{m}$. Flagellomere lengths (in $\mu \mathrm{m}$ ): $37.5 ; 17.5 ; 15.0 ; 2.5 ; 2.5 ; 2.5$; AR 0.90; first segment 3.0 times as long as basal width, ring organ located near the middle area, 0.56 times first segment from the base. Blade $70 \mu \mathrm{m}$ long, 1.56 times as long as flagellum. Style small, $2 \mu \mathrm{m}$ long, located at the apex of third segment.

Labro-epipharynx: SI plumose on distal margin (Figure 5B), without indication of fusion in the base. Distal margin of labral lamella with 15 teeth. Pecten epipharyngis consists of 3 scales, each with 3 small teeth. Premandible (Figure 5C) $40 \mu \mathrm{m}$ long, two inner teeth close to two apical teeth, premandibular brush absent.

Mandible (Figure 5D): Total length $130 \mu \mathrm{m}$, with two small inner teeth and one apical tooth. Apical tooth predominant, 80 $\mu \mathrm{m}$ long, about the same as the basal part. Seta subdentalis 48 $\mu \mathrm{m}$ long, extending along the inner margin of apical tooth.

Mentum (Figure 5E): $65 \mu \mathrm{m}$ wide, median cleft depth $62.5 \mu \mathrm{m}$, each with 8 lateral teeth, inner most one pair teeth paler than others. Ventromentum $164 \mu \mathrm{m}$ wide, ventromentum plate ratio 4.0. Inter-plates distance $26 \mu \mathrm{m}$ long. Setae submenti posterior to the mentum, distance between setae submenti $90 \mu \mathrm{m}$.

Body: Procercus squat, $45 \mu \mathrm{m}$ wide, $30 \mu \mathrm{m}$ high. Anal setae not clear. Upper anal tubules $135 \mu \mathrm{m}$ long, 2.8 times as long as basal width; lower pairs $165 \mu \mathrm{m}$ long, 3.2 times as long as basal width.

\section{"Nilodosis" sp. Yunnan}

(Figure 6)

Material examined. 1 larva, China, Yunnan Province, Xishuangbanna, Langcang River (upstream of Mekong River), 15. vii. $2010\left(22^{\circ} 04^{\prime} 12.26^{\prime \prime} \mathrm{N}, 100^{\circ} 45^{\prime} 24.99^{\prime \prime}\right.$ E), leg. Y. Jiang.

Total length $10.6 \mathrm{~mm}$. Head capsule yellow brown, $560 \mu \mathrm{m}$ long, $355 \mu \mathrm{m}$ wide. Postmentum length $285 \mu \mathrm{m}$.

Antenna (Figure 6A): Total length 115-120 $\mu \mathrm{m}$. Flagellomere lengths (in $\mu \mathrm{m}$ ): $50.0-52.5 ; 37.5-40.0 ; 12.5-15.0 ; 2.5 ; 5 ; 2.5 ; \mathrm{AR}$ 0.75-0.77; first segment 3.0-3.3 times as long as basal width, ring organ located near the middle area, 0.53-0.60 times first segment from the base. Blade 75-80 $\mu \mathrm{m}$ long, 1.23-1.25 times
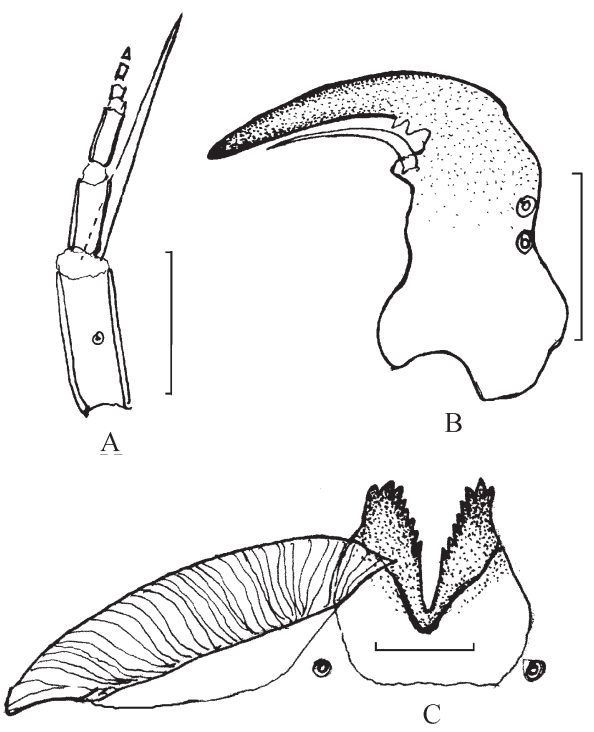

Figure 6. Larval morphology of "Nilodosis" sp. Yunnan. A) Antenna; B) Mandible; C) Mentum (scale $=50 \mu \mathrm{m}$ ).

as long as flagellum. Style apparently absent.

Labro-epipharynx: SI plumose on distal margin. Pecten epipharyngis not observable. Premandible as in generic diagnosis with 4 teeth.

Mandible (Figure 6B): Total length 150-160 $\mu \mathrm{m}$, with two small inner teeth and one apical tooth which 95-100 $\mu \mathrm{m}$ long, about the same as the basal part. Seta subdentalis not apparent.

Mentum (Figure 6C): $65 \mu \mathrm{m}$ wide, medial cleft depth $60 \mu \mathrm{m}$, each side with 8 lateral teeth, innermost mental teeth the same color as other laterals. Ventromentum $160 \mu \mathrm{m}$ wide about 4.0 times as long as height. Inter-plates distance $25 \mu \mathrm{m}$ long. Setae submenti posterior to the mentum, distance between setae submenti $100 \mu \mathrm{m}$.

Body: Procercus higher than wide, about 2 times as long as width. 6 anal setae, 500-600 $\mu \mathrm{m}$ long, supraanal seta 400-450 $\mu \mathrm{m}$ long. Anal tubules 200-250 $\mu \mathrm{m}$ long, about 4.5-5.0 times as long as basal width.

\section{“Nilodosis" sp. Japan}

(Figures 7-11)

Chironominae Genus CA, Kitagawa: 1997: 165.

Fissimentum sp FA, Kitagawa: 2001: 3.

Fissimentum sp., Ueno et al. 2001: 100.

Material examined. 2 larvae, Japan, Aichi Prefecture, Noubi Bridge, Kiso River, 23. vi. 1997, leg. N. Kitagawa; 40 larvae, as previous except, Sobue, Kiso River, 24. i, 2007, leg. G. Yoshinari; 25 larvae, as previous except 16. ii. 2007; 2 larvae, Japan, Shiga Prefecture, Yasu City, Yasu River, inlet of Lake Biwa, 6-7. vi. 1997, leg. N. Kitagawa; 1 larva, Japan, Yamanashi Prefecture, Fuji-Kawaguchi, Lake Motosu, 20. vii. 2007, leg. N. Kitagawa.

Description ( $\mathrm{n}=11$, unless otherwise stated) 
Live larvae reddish (Kitagawa, Ueno and Yoshinari, pers comm.), total length $12-13 \mathrm{~mm}$. Head capsule brown, with a distinct dark brown triangulum occipitale (Figure 7A-C, 8A). Head length 574-681, $628 \mu \mathrm{m}$ long; head width 279-361, $312 \mu \mathrm{m}$ long $(\mathrm{n}=7)$. Head length/head width 1.89-2.18, 1.99 .

Dorsal surface (Figure 7A) and chaetotaxy of head capsule (Figure 7A-C): Frons about 1/2 as long as head capsule length, with anterior margin nearly straight, without fenestra. Clypeal sclerite entirely fragmented into tiny particles, labral sclerite complete. Frontal seta S4 elongate. Suborbital seta (S6) and supraorbital seta (S7) adjacent to each other; S7 and genal seta 9 (S9) rather short and distinctly expanded apically, club- to balloon-like (Figure 8B).

Antenna (Figure 8C; n=7): AR 0.80-1.09, 0.94. Antennal segment lengths (in $\mu \mathrm{m}$ ): 54-70, 60; 26-30, 29; 8-20, 14; 4-5, 6; 4-6, 5. Blade (n=4) 1.8 times as long as basal segment, 80-96, $85 \mu \mathrm{m}$ long. Style at third segment $(\mathrm{n}=6)$, reaching to the base of 4th segment, 7-10, $8 \mu \mathrm{m}$ long.

Labro-epipharynx (Figure 9A): SI broad, strongly expanded
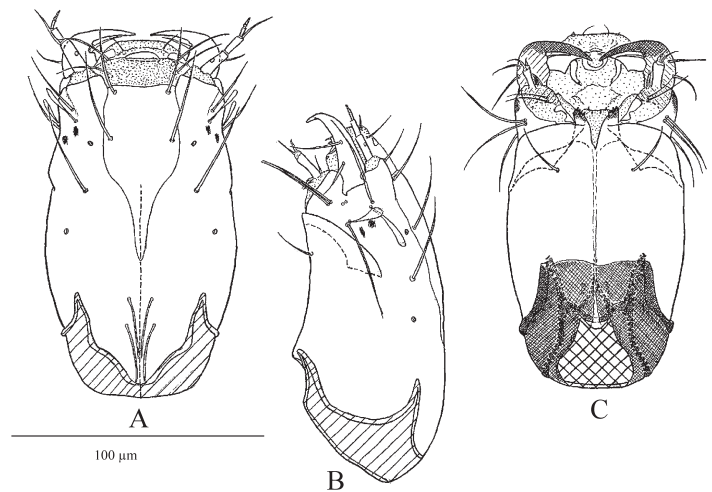

Figure 7. Head capsule and cephalic setation of "Nilodosis" sp. Japan. A) Dorsal view; B) Lateral view; C) Ventral view.

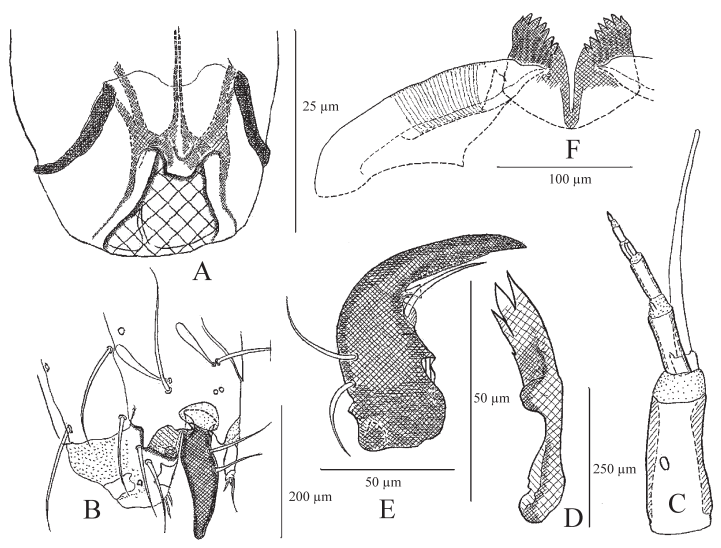

Figure 8. A) Ventral view of posterior part of head capsule "Nilodosis" sp. Japan, indicating the contact line between head wall and somatic membrane and posterior tentorial pit; B) Lateral view of dorsal surface of head and cephalic setation; C) Antenna; D) Premandible; E) Mandible; F) Mentum. distally, plumose on distal margin. Base of SI contiguous. Both S II and S III slender and simple. Two pairs of distinct spinulae weakly scleotized, triangular, scale like. Two pairs of labral chaetae rather long, serrated apically. Labral lamellae broad, strongly sclerotized, without indication of median division, with 9-18 nearly even teeth distally. Pecten epipharyngis of one small plate, apically serrated. Chaetulae laterals with 4-5 pairs, each with many branches on its apical portion; chaetulae basales 3-4 pairs, anterior 2 serrated apically. Premandible (Figure 8D) with 4 pointed teeth, basal one tiny, 42-64, $55 \mu \mathrm{m}$ long. Premadibular brush absent.

Mandible (Figure 8E; $\mathrm{n}=8$ ): With one distinct apically expanded

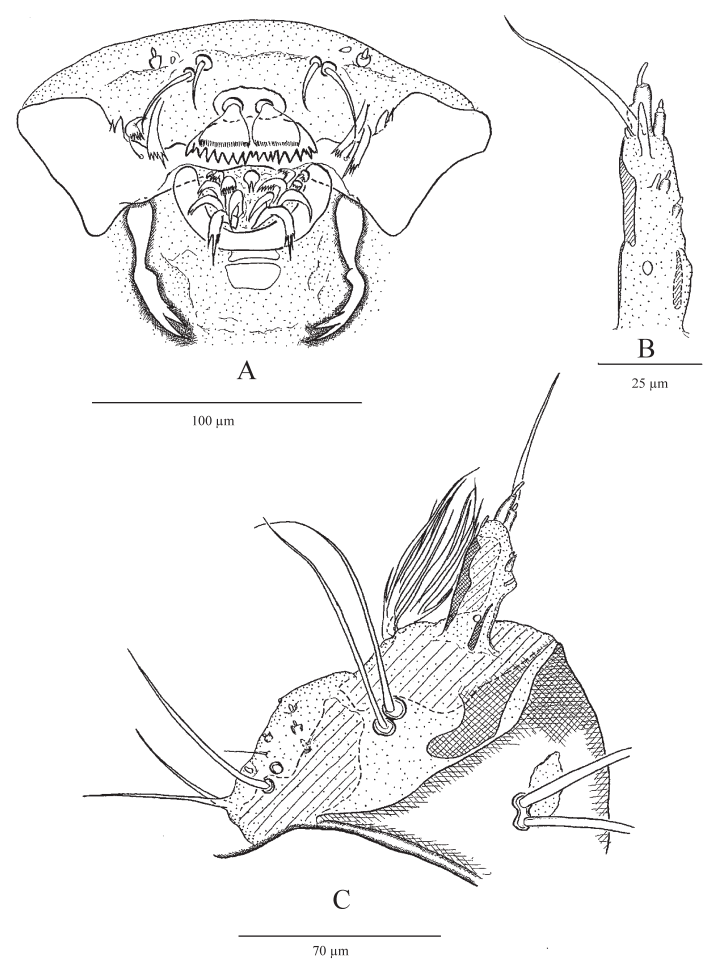

Figure 9. Larval morphology of "Nilodosis" sp. Japan. A) Labroepipharynis; B) Details of maxillary palp; C) Maxilla.

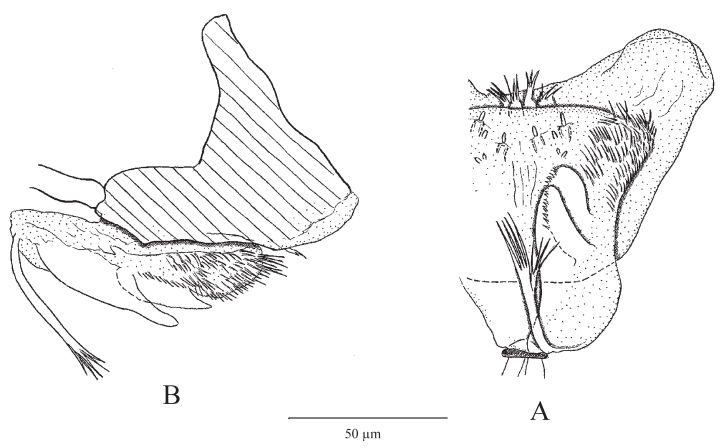

Figure 10. Larval prementum and hypopharynx of "Nilodosis" sp. Japan. 
and truncated thin blade-like large inner tooth, together with 1-3 additional associated small teeth. Seta subdentalis $56-74$, $66 \mu \mathrm{m}$ long.

Maxilla (Figure 9B, C): Lacinial chaetae being 2 hyaline thin and slender simple blades, 34-62, $52 \mu \mathrm{m}$ long $(\mathrm{n}=6)$. Antaxial seta long, 76-104, $87 \mu \mathrm{m}$ long $(\mathrm{n}=9)$, paraxial setae short, setiform, 8-14, $13 \mu \mathrm{m}$ long $(\mathrm{n}=6$ ). Palpiger (Figure 9B) with 2 close-set chaetulae, each of which is multiply forked at base. Maxillary palp 54-66, $60 \mu \mathrm{m}$ long, apical setae about $60 \mu \mathrm{m}$ long, ring organ situated at middle.

Mentum and ventromental plate (Figure 8F) as in generic diagnosis. Each side of mentum with 8 teeth.

Prementum (Figure 10A, B): Anterior margin smoothly rounded, without any distinct structures. Simple chaetulae present apicolaterally. Five pairs of sensilla basiconica distinctly

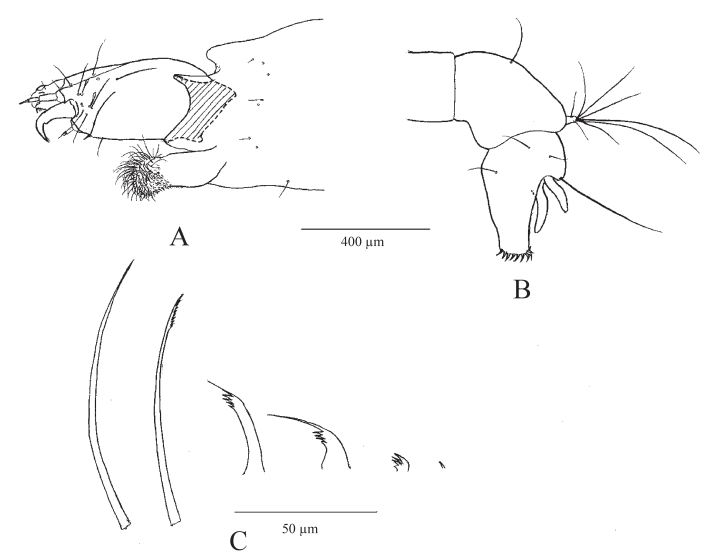

Figure II. Abdominal characters of "Nilodosis" sp. Japan. A) Anterior parapods; B) Body posterior part; C) Anterior parapod claws.

Table 2. The top 5 abundant co-inhabited chironomids with Nilodosis larva at different sampling sites (for "Nilodosis" sp. Japan, the community data adapted from Ueno et al., 2001).

\begin{tabular}{lllll}
\hline Community & Korea & China (Guangdong) & China (Yunnan) & Japan \\
\hline TOP1 & Alabesmyia monilis & Tanytarsus cf. ejuncidus & Polypedilum cultellatum & Procladius sp. \\
TOP2 & Tanytarsus oscillans & Polypedilum cultellatum & Tanytarsus mendax & Stictochironomus sp. \\
TOP3 & Polypedilum nubeculosum & Polypedilum nubeculosum & Cricotopus trifascia & Ablabesmyia sp. \\
TOP4 & Glyptotendipes tokunagai & Kiefferulus barbatitarsis & Rheocricotopus fucipes & Tanytarsus sp. \& Polypedilum sp. \\
TOP5 & Cryptochironomus rostratus & Microchironomus tener & Nanocladius sp. & Cryptochironomus sp. \\
\hline
\end{tabular}

Table 3. The associated environmental parameters at the two sampling sites, including the concentration of dissolved oxygen (DO), biological oxygen demands (BOD), chemical oxygen demands (COD), total nitrogen (TN), total phosphorus (TP) and chlorophyll a (Chla). Units of Chl-a is $\mu \mathrm{g} / \mathrm{L}$, while the unit of others are all $\mathrm{mg} / \mathrm{L}$.

\begin{tabular}{lcccccc}
\hline Sites & DO & BOD & COD & TN & TP & Chl-a \\
\hline China (Guangzhou) & $7.00-7.80$ & $3.25-4.00$ & $2.00-2.50$ & $0.48-0.69$ & $0.006-0.016$ & $2.66-4.53$ \\
Korea (Jinyang) & $7.57-8.79$ & $1.99-2.33$ & $4.47-5.58$ & $0.75-0.76$ & $0.015-0.027$ & $8.90-21.40$ \\
\hline
\end{tabular}

Table 4. A comparison of morphological measurements between 4 different larval forms of "Nilodosis" (The unit is $\mu \mathrm{m}$, "-." denotes missing values, all abbreviations follows Sæther, 1980 and Sæther et al., 2000).

\begin{tabular}{lcccc}
\hline Parameters & sp. Korea & sp. Guangdong & sp. Yunnan & sp. Japan \\
\hline Head L & 630 & 500 & 560 & 628 \\
Head W & 365 & 300 & 355 & 312 \\
Postmentum & 418 & 233 & 285 & - \\
SSm-SSm & 145 & 90 & 100 & - \\
An & 135 & 75 & 118 & 120 \\
AR & 0.83 & 0.9 & 0.76 & 0.94 \\
A1 & 61 & 37.5 & 51 & 60 \\
BL & 93 & 70 & 78 & 85 \\
BL/FL & 1.28 & 1.56 & 1.24 & 1.37 \\
VmP & 208 & 164 & 160 & - \\
IPD & 48 & 26 & 25 & - \\
Mentum width & 87 & 65 & 65 & - \\
Total length $(\mathrm{mm})$ & 11.3 & 8.75 & 10.6 & 12.5 \\
\hline
\end{tabular}


small compared with those of Chironomus, Einfeldia and Glyptotendipes. Two pairs of rather wide and thin blades present mediolaterally, anterior pair of which are serrated on apical margins. One pair of long and slender appendages originate on the caudal area.

Hypopharynx (Figure 10A, B): Anterolateral corner strongly expanded, with two pairs of apically trifid appendages medially.

Body (Figure 11A-C): Anterior parapods with dense, fine claws, some finely or distinctly serrated apically. Procercus 2.0 times as long as wide, bearing 6-7, 7 subequal anal setae. Supraanal seta as long as anal seta. Two pair of anal tubules nearly equal sized.

Remarks. In his drawings, Kitagawa (1997) failed to recognize the split between S4 and S5 on the dorsal head as a suture, the posterior margin of the labral lamella as distal margins of the SI setae and the thin apically truncated inner tooth of the mandible as a simple triangular tooth. We found no morphological differences between Kitagawa's specimens and our own specimens and concluded that these specimens are conspecific. Although they share the club- to balloon-shaped S7 and S9, "Nilodosis" sp. Japan is separated from "Nilodosis" sp. Korea by the shape of the inner mandibular and premandibular teeth. "Nilodosis" sp. Japan is distinct from the African Nilodosis fusca in the following combination of characters: Frontal seta S4 elongate; labral lamella wide, strongly sclerotized and with about 20 nearly even teeth on the distal margin; premandible with 4 teeth and without brush; pecten epipharyngis consisting of 1 apically toothed scale; lacinial chaeta long and slender. The species resembles Fissimentum sp. 2 of Cranston and Nolte (1996) from Brazil in general appearance of head capsule.

\section{Ecology and distribution}

Most sampling sites in Asia indicate that the larvae can occur in both the littoral zone of lakes (Jinyang Lake in Korea; Biwa Lake, Motosu Lake, Towada Lake in Japan) and in the potamals of larger rivers with maximum depth no more than 5 meters (Kiso River, downstream of Noubi bridge, Aichi Prefecture (Kitagawa, 1997); Mekong River, China). Several sampling sites were in the riverine zone of a reservoir (Luitian River, China) or main tributaries flowing to the lakes (Yasu River, inlet of Biwa Lake, Shiga Prefecture, Japan (Kitagawa, 1997)). The substrata consisted of mud-sand fine particles and a layer of silt and organic matter in the upper surface. Community structure shows that the co-inhabited species generally includes herbivores, shredders and Harnischia complex predators in the top five dominant species (Table 2). Based on the cleft mentum and strong curved mandible, the larva is probably a semipsammorheophilic predator.

The associated environmental parameters were also measured in Korea and China Guangdong sites (Table 3). Generally, larvae prefer relative clean larger water bodies, ranging from the mesotrophic to the eutrophic zone.

\section{DISCUSSION}

Cephalic setae of variable forms are found in the genus. "Nilodosis" sp. Japan bears club- to balloon-shaped S7 and S9; these are observed also in the Korean material. The close gap between S6 and S7 was confirmed in Korean material. The cephalic setae in "Nilodosis" sp. Guangdong and "Nilodosis" sp. Yunnan are hard to observe and some setae were lost during slide mounting. The frontal seta $\mathrm{S} 4$ is reduced in $N$. fusca, while this feature was not observed in all larvae examined in this study. The long-curved mandible may be associated with its predatory behavior, which can be observed also in some Tanypodinae genera (e.g., Clinotanypus, Psectrotanypus) and some genera of the Harnischia-complex (e.g. Chernovskiia, Cryptochironomus, Demicryptochironomus and Robackia). The arrangement of the mandible inner teeth is probably also related to the feeding behavior. The three clumped inner teeth of N. fusca, found also in the genera Nilothauma and Pagastia, make a tight fit, preventing the collected food from escaping easily. All the Asian larval forms described in this paper bear one distinct large tooth together with 1-3 small teeth, which is very commonly found in the genera of subfamily Tanypodinae, and may assist in holding the captured prey tightly. Therefore, the characters of long-curved mandible and inner teeth in the predacious chironomid larvae are due to convergent evolution and not homologous characters. The maxilla bears two distinct cluster of chaetulae dorsal to the palpiger, and the seta of the maxillary palp is well developed. These characters differ from the reduced maxilla found in larvae of Fissimentum.

Until the Asian immature stages are associated with the adults generic assignment to Nilodosis, and species delimitation remain tentative. We still find it valuable that the larval forms are described. Based on comparative measurements and morphology (Table 4), the Japanese larval form is most similar to the Korean larval form. The Chinese Guandong and Yunnan larval forms differ from the above, and may represent different species. Guangdong larval forms can be separated from others by the subequal length of the antennal segments 2 and 3; and Yunnan larval form can be separated from others by having the same color on all mental teeth. More detailed morphological analysis of associated pupae and adults of the above described larval forms is required for a precise generic diagnostics of Nilodosis.

\section{ACKNOWLEDGMENTS}

This study was financially supported by the "Fundamental Research Funds for the Central Universities, 2010" (No. $11609320,2010)$ and "the projects for developing excellent young creative talents in Guangdong universities (cultivation project for youth) grant funded by Guangdong Government “ (No. 34310015, 2011-2012).

The senior author is indebted to Professor Peter Cranston, 
Australian National University, Canberra, Australia, for discussion of the genus Nilodosis at the $18^{\text {th }}$ Chironomidae symposium conference, Trondheim, Norway. Special thanks to Mr. Norizumi Kitagawa, Mie Prefecture, for sending all of his publications and loan of his materials, and to Mr. Gyo Yoshinari, Shizuoka Prefecture, for offering us invaluable specimens. Mr. Woon-Seok Cho and Yang Jiang provided the materials and some environmental data. Many thanks to Prof. X.H. Wang, Nankai University, Tianjin, China and Tae-Soo Chon, Pusan National University, Pusan, South Korea for providing a comfortable scientific atmosphere during my $\mathrm{PhD}$ and post-doctoral work.

\section{REFERENCES}

Chrispeels A. 1959. Larves de Chironomidae (Diptera Nematocera). Résultats Scientifiques. Exploration Hydrobiologique des Lacs Kivu, Édouard et Albert 3: 140-188.

Cranston PS, Nolte U. 1996. Fissimentum, a new genus of drought-tolerant chironomini (Diptera: Chironomidae) from the Americas and Australia. Entomological News 107: 1-15.

Cranston PS, Spies M. 1999. The immature stages and phylogenetic position of Nilodosis Kieffer, an Afrotropical genus of Chironomini (Diptera: Chironomidae). African Entomology 7: 249-259.

Eggermont H, Verschuren D. 2003. Subfossil Chironomidae from Lake Tanganyika, East Africa 2. Chironominae (Chironomini and Tanytarsini). Journal of Paleolimnology 29: 423-458.

Epler JH. 2001. Identification Manual for the larval Chironomidae (Diptera) of North and South Carolina. A guide to the taxonomy of the midges of the southeastern United States, including Florida. Special Publication SJ2001-SP13. North Carolina Department of Environment and Natural Resources, Raleigh, NC, and St. Johns River Water Management District, Palatka, FL. 526 pp.

Freeman P. 1957a. Chironomidae (Diptera, Nematocera). Imagines. Résultats Scientifiques. Exploration Hydrobiologique des Lacs Kivu, Édouard et Albert 3: 205-221.

Freeman P. 1957b. A study of the Chironomidae (Diptera) of Africa south of the Sahara. Part III. Bulletin of the British Museum Natural History (Entomology) 5: 323-426.

Goetghebuer M. 1936. Chironomides du Congo Belge. Revue de Zoologie et de Botanique Africaine 28:453-492.

Hare L, Carter JCE. 1987. Chironomidae (Diptera, Insecta) from the environs of a natural West African lake. Entomologica Scandinavica Supplement 29: 65-74.

Kieffer JJ. 1921. Chironomides de l'Afrique Equatoriale (1re partie). Annales de la Société Entomologique de France 90: 1-56.

Kitagawa N. 1997. Chironomid larvae from Kiso River - twenty species of larvae collected at near Nohbi Ohashi Bridge. Tansuiseibutsu (Freshwater Biology), 74: 77-99 (in Japanese).

Kitagawa N. 2001. Taxonomic studies on Chironomid larvae (3). Tansuiseibutsu (Freshwater Biology), 81: 1-7 (in Japanese).

McLachlan AJ. 1969. Notes on some larval and pupal chironomids (Diptera) from Lake Kariba, Rhodesia. Journal of Natural History 3: 261-293.
Pinder LCV, Reiss F. 1983. The larvae of Chironominae (Diptera: Chironomidae) of the Holarctic region. Keys and diagnoses. Entomologica Scandinavica, Supplement 19: 293-435.

Sæther OA. 1980. Glossary of chironomid morphology terminology (Diptera: Chironomidae). Entomologica Scandinavica, Supplement 16: 1-51.

Sæther OA. 2000. Zoogeographical patterns in Chironomidae (Diptera). Verhandlungen der Internationalen Vereinuigung für Theoretische und Angewandte Limnologie 27: 209-302.

Sæther OA, Ashe P, Murray DE. 2000. Family Chironomidae. In: Papp L, and Darvas B (eds). Contributions to a Manual of Palaearctic Diptera (with special reference to the flies of economic importance). Vol. 4. Appendix A.6. Science Herald, Budapest, pp.113-334

Ueno R, Nohara S, Kato H. 2001. Distribution of chironomids in the littoral zone of lake Towada. Research Report of National Institute Environment Study, Japan (167): 99-101.

Verschuren D, Eggermont H. 2006. Quaternary paleoecology of aquatic Diptera in tropical and Southern Hemisphere regions, with special reference to the Chironomidae. Quaternary Science Reviews 25: 1926-1947. 